Supporting Information

\title{
Mapping the Space Charge at Nanoscale in Dielectric Polymer Nanocomposites
}

Simin Peng, Zhen Luo, Shaojie Wang, Jiajie Liang, Chao Yuan, Zhikang, Yuan, Jun $\mathrm{Hu}^{*}$, Jinliang $\mathrm{He}^{*}$, and Qi Li*

State Key Laboratory of Power System, Department of Electrical Engineering,

Tsinghua University, Beijing 100084, China

Email: qili1020@,tsinghua.edu.cn (Q.L.); hej1@,tsinghua.edu.cn (J.L.H.);

hjun@tsinghua.edu.cn (J.H.) 


\section{Supplementary Note 1:}

The vibration of the probe can be described as a forced Lorentz oscillator model:

$$
m \ddot{x}+\frac{m \omega_{0}}{Q} \dot{x}+k x=F_{\mathrm{d}} \cos \omega t
$$

where $m, Q$ and $k$ are the mass, quality factor and elastic coefficient of the probe cantilever, respectively, $x$ and $\omega_{0}$ are the vibration displacement and resonance angular frequency of the probe cantilever, respectively, and $\omega_{0}=\sqrt{k / m}, F_{\mathrm{d}}$ and $\omega$ are the amplitude and angular frequency of driving force applied on the tip. Since van der Waals force is ignored, the driving force is mainly the electrostatic force. The vibration displacement of the probe cantilever can be solved as

$$
x=B e^{-\alpha t} \cos \left(\omega_{\mathrm{r}} t+\beta\right)+A \cos (\omega t-\phi)
$$

the steady-state solution is

$$
\begin{gathered}
x=A \cos (\omega t-\phi) \\
A=\frac{\left|F_{\mathrm{d}}\right|}{m \sqrt{\left(\frac{\omega_{0} \omega}{Q}\right)^{2}+\left(\omega_{0}^{2}-\omega^{2}\right)^{2}}}
\end{gathered}
$$

\section{Supplementary Note 2:}

In order to facilitate the analysis, we chose the $A_{2 \omega}$ data along the horizontal line in the middle of the micro-area in each figure shown in Figure S1. Here several conclusions can be drawn from the results. First, the results reveal that the $A_{2 \omega}$ signals of the nanoparticles are significantly lower than that of the rest micro-area, and the latter is almost a constant value, as shown in the curves in Figure S1, although the topography of polymer matrix has some fluctuations (about $20 \mathrm{~nm}$ ). According to Eq. 8 in the main text, since $A_{2 \omega}$ is proportional to $\partial C / \partial z$, it is easy to draw the conclusion that $\partial C / \partial z$ is hardly affected by the thickness of the film. On the other hand, during the three phases (intrinsic phase, voltage-applying phase and recovery phase), $A_{2 \omega}$ signals also remain unchanged, which demonstrates that the $\partial C / \partial z$ is also independent of the applied lateral electric field. 


\section{Supplementary Note 3:}

The surface potential difference signal $\Delta V$ is considered to be related to the work function difference (noted as $\Delta \Phi$ ) between the sample and the tip, the contribution of free surface charges and orientation dipole moment of the sample (noted as $\mu$ ), and the contribution of the rearrangement of electrons from the sample/substrate interface (noted as $\delta$ )[8]. In this study, the contribution of free surface charges is discussed separately. Aside from the free charges, the sample's electronic structure is relatively simple and $\delta$ can be ignored, and there is no induced polarization in the vertical direction because no vertical DC voltage is applied to the tip. Therefore, $\Delta V$ can be considered to be only related to the work function difference $(\Delta \Phi)$ between the sample and the tip

\section{Supplementary Note 4:}

When the lateral electric field strength is low, the charge injection from the electrode is not significant, and the amount of charges trapped by the interfacial traps is quite low. Therefore, the local $C E_{\mathrm{z}}$ signal generated by trapped charges is weak, which is masked by that generated by charges on the surface of the matrix. By contrast, when the lateral electric field strength is high, the amount of charges trapped by the interfacial traps becomes large, and the local $C E_{\mathrm{z}}$ signal generated by trapped charges is strong. Another question is the difference in $V_{\text {flatten }}$ signals under positive and negative lateral electric fields. In the experimental system, the electrode on the right side of the test micro-area is grounded, since the majority carriers of $\mathrm{P}(\mathrm{VDF}-\mathrm{TrFE})$ are electrons, when the negative voltage is applied to the left electrode, electrons are injected into the sample, which makes the number of electrons participating in charge transport increases, while when the positive voltage is applied to the left electrode, electrons are extracted from the sample, which makes the number of electrons participating in charge transport decreases. As a result, under the positive lateral electric field, no significant signal of trapped charges at the interface is observed in the polymer nanocomposite (Figure S3). 


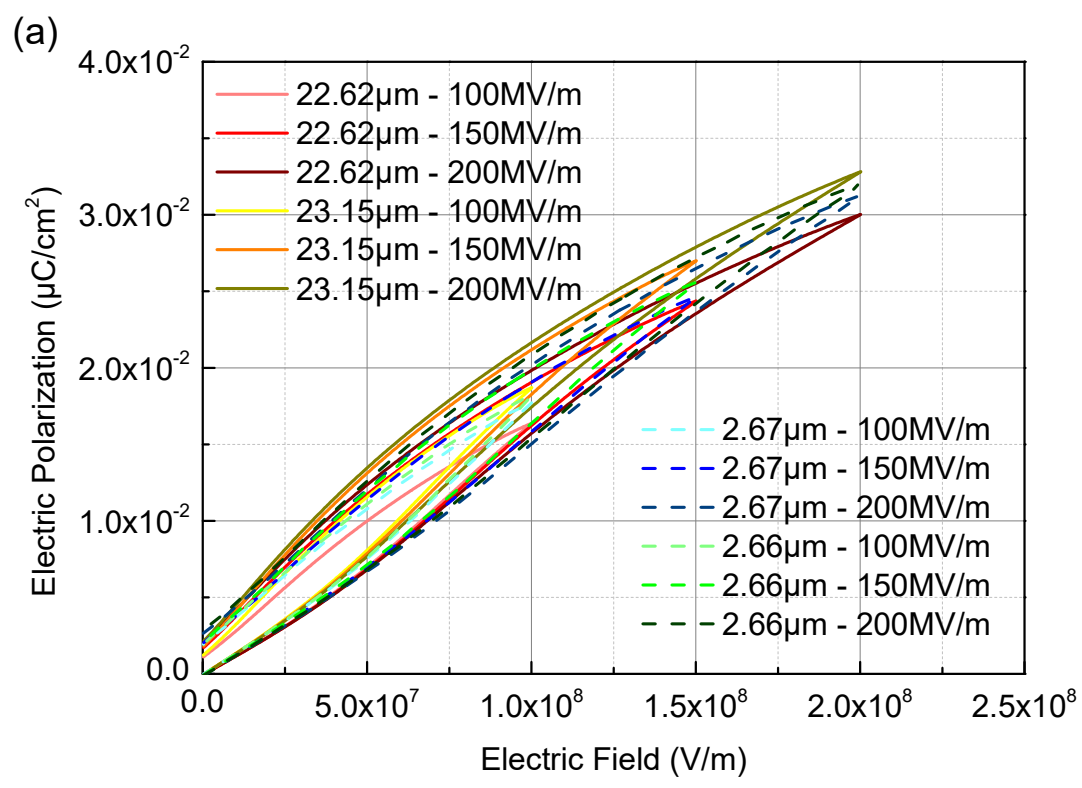

(b)

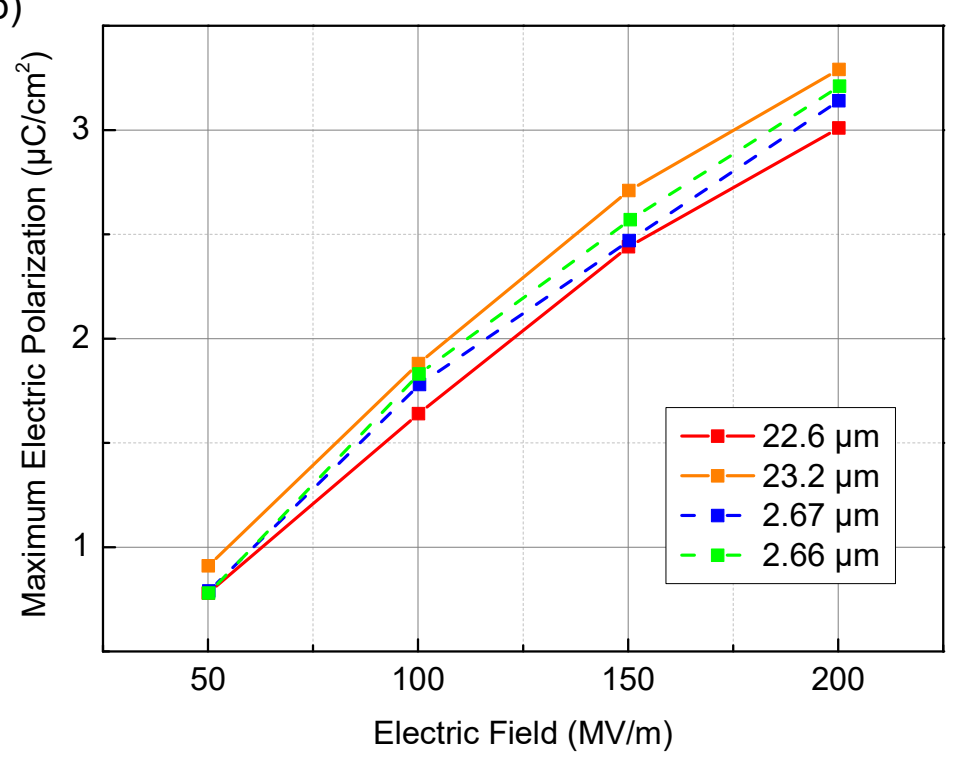

Figure S1 (a) Electric polarization-electric field loops and (b) maximum electric polarization of the thin and thick films of $\mathrm{P}(\mathrm{VDF}-\mathrm{TrFE})$. It is apparent that the films with different thickness are very similar in both the loop shape and the amplitude of maximum electric polarization. This result suggests that there is negligible electric field drop at the electrode/polymer interface. This is because if there is significant electric field drop at the electrode/polymer interface when the film is thin, the electric polarization of the thin and thick films will be very different due to the fact that the electric polarization of the material is proportional to the electric field applied on it. 


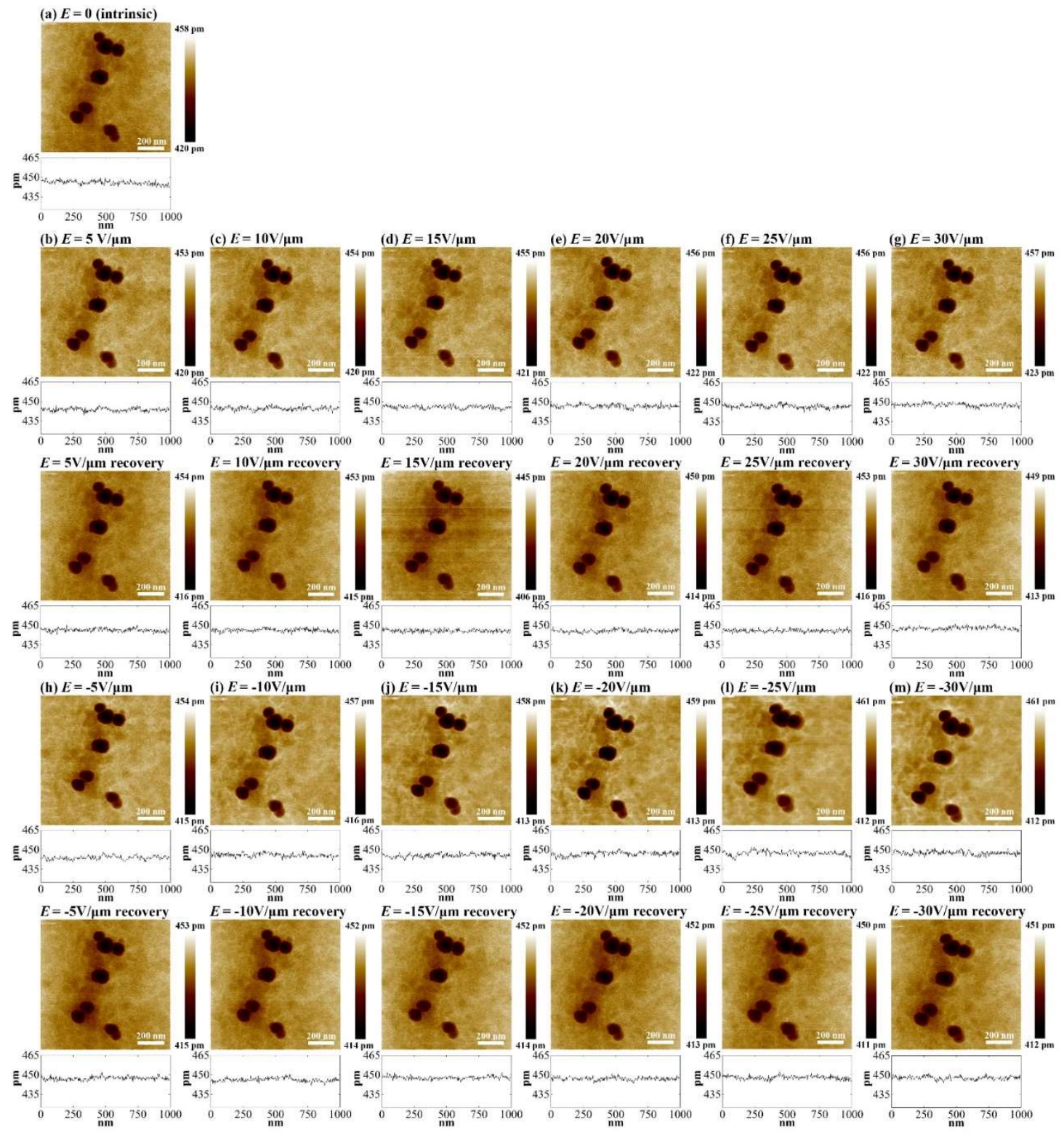

Figure S2 $A_{2 \omega}$ signals (a) with and (b-m) without the lateral electric field. The nanoparticles are more than $70 \mathrm{~nm}$ higher than that of the polymer matrix, representing a major topography fluctuation. In this case, $\partial \mathrm{C} / \partial \mathrm{z}$ measured on the nanoparticles is not directly proportional to the dielectric constant. 


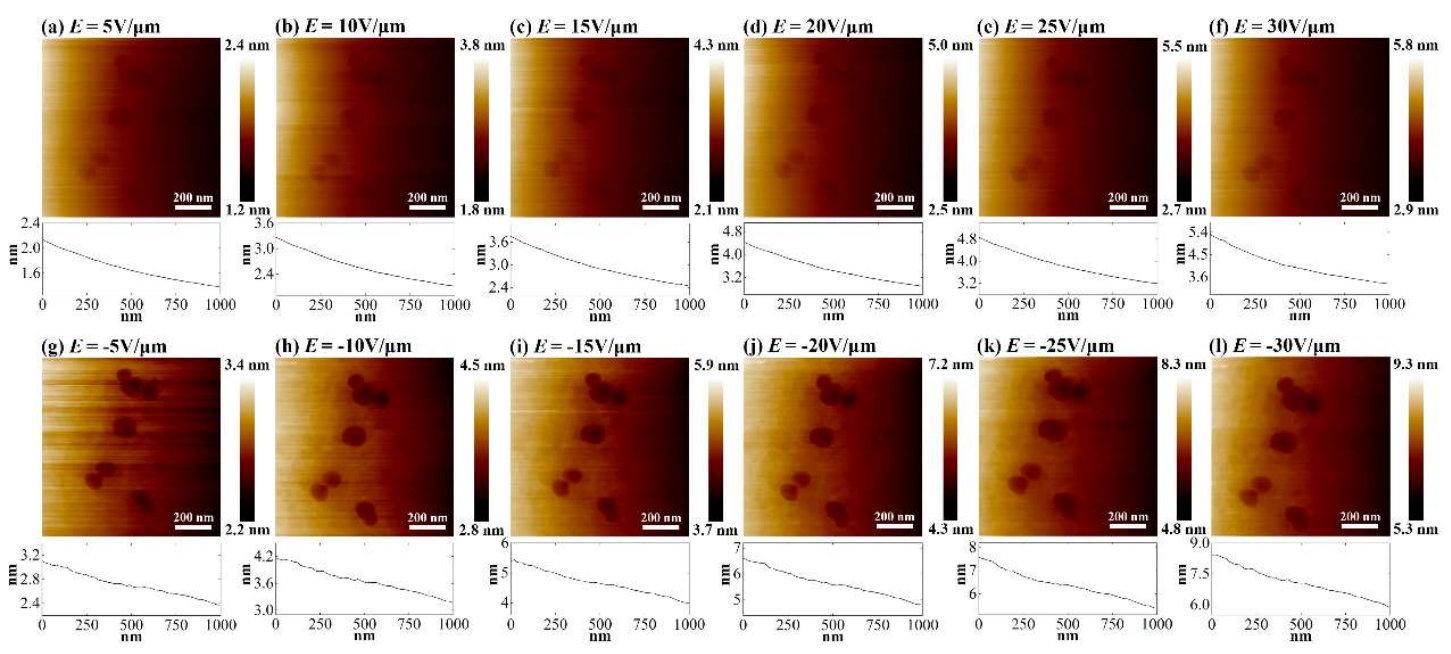

Figure S3 The results of $A_{\omega}$ with the applied (a-f) positive and (g-l) negative lateral electric fields. 

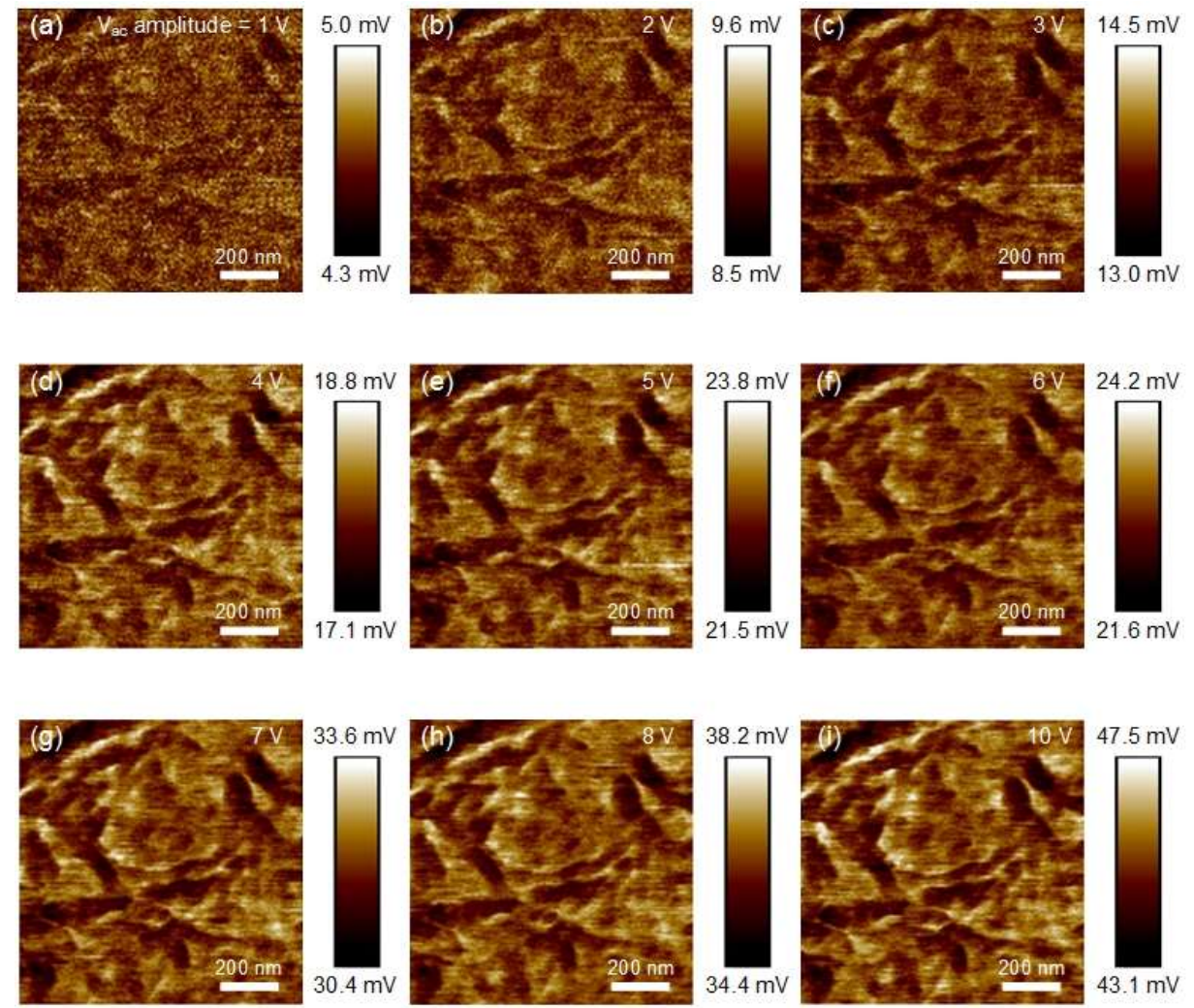

Figure S4 (a-i) Mapping of $A_{\omega}$ at the $\mathrm{AC}$ amplitude ranging from $1 \mathrm{~V}$ to $10 \mathrm{~V}$. 

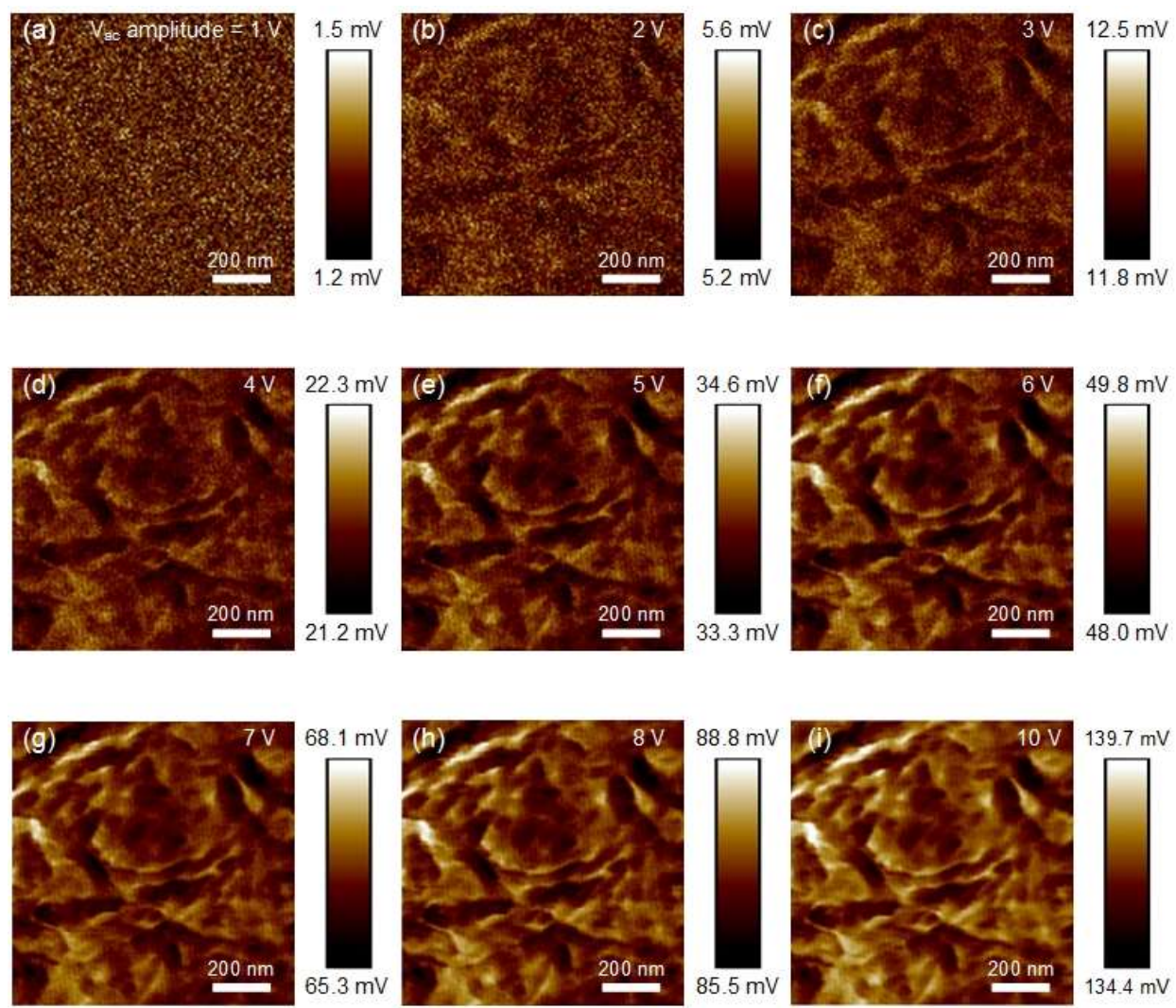

Figure S5 (a-i) Mapping of $A_{2 \omega}$ at the $\mathrm{AC}$ amplitude ranging from $1 \mathrm{~V}$ to $10 \mathrm{~V}$. 
(a)

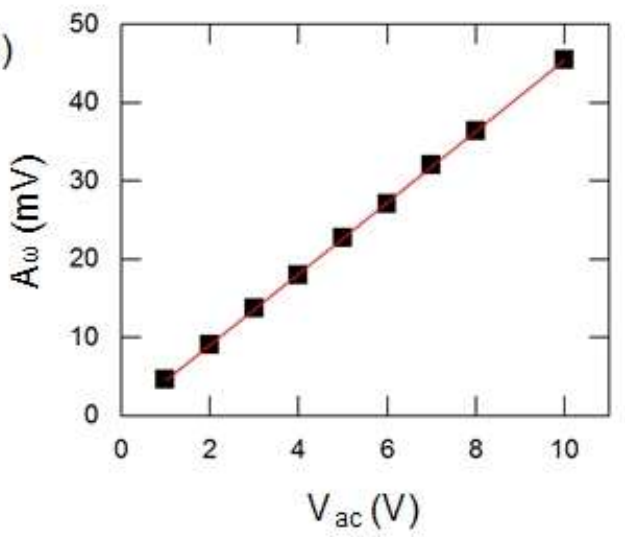

(b)

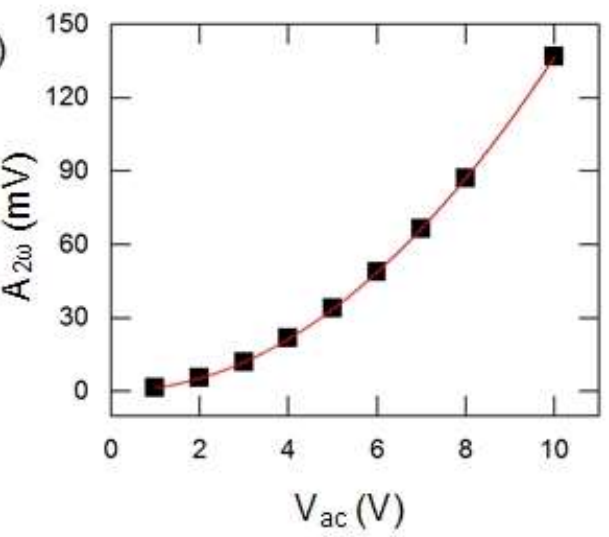

Figure S6 The dependence of (a) $A_{\omega}$ and (b) $A_{2 \omega}$ on the AC amplitude 
(a) $E=0$

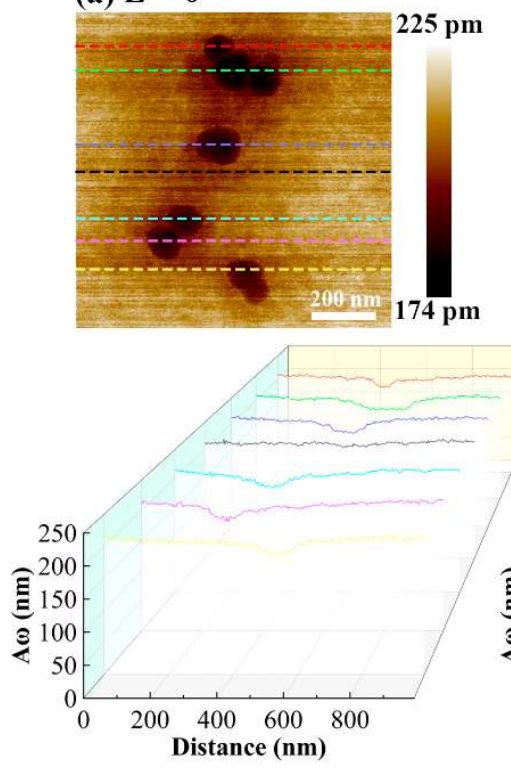

(b) $E=5 \mathrm{~V} / \mu \mathrm{m}$

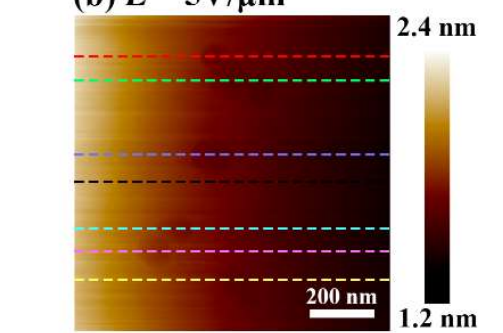

(c) $E=-5 \mathrm{~V} / \mu \mathrm{m}$
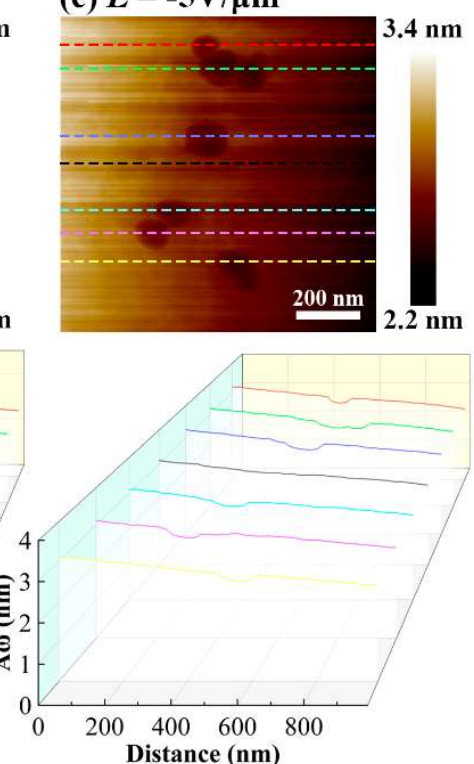

Figure S7 (a) The $A_{\omega}$ signal without the applied lateral electric field. (b, c) The $A_{\omega}$ signals with $5 \mathrm{~V} / \mu \mathrm{m}$ and $-5 \mathrm{~V} / \mu \mathrm{m}$ applied lateral electric field, respectively. 
(a) $E=5 \mathrm{~V} / \mu \mathrm{m}$

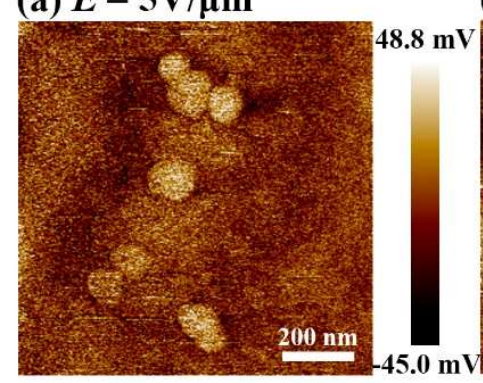

(d) $E=20 \mathrm{~V} / \mu \mathrm{m}$

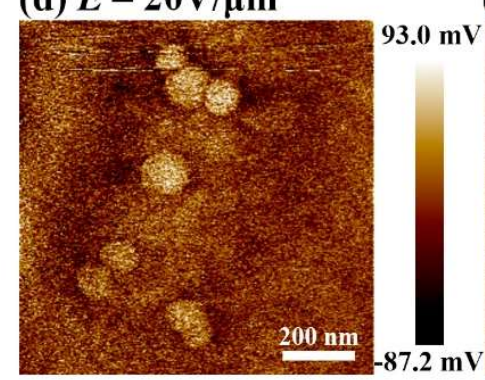

(b) $E=10 \mathrm{~V} / \mu \mathrm{m}$

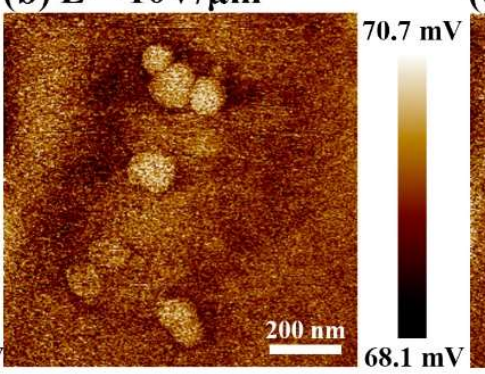

(c) $E=15 \mathrm{~V} / \mu \mathrm{m}$

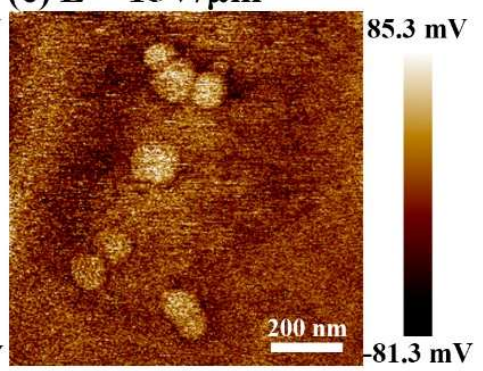

(e) $E=25 \mathrm{~V} / \mu \mathrm{m}$

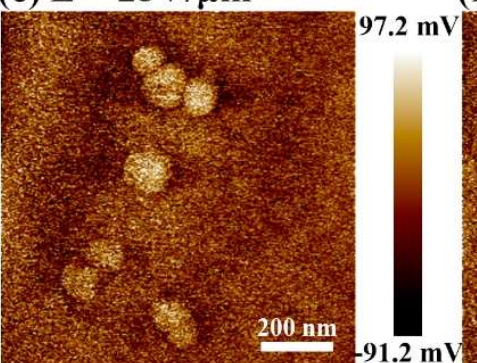

(f) $E=30 \mathrm{~V} / \mu \mathrm{m}$

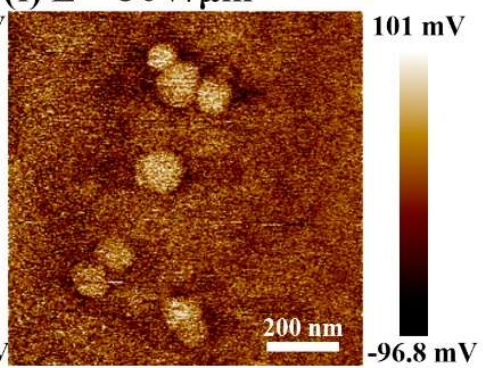

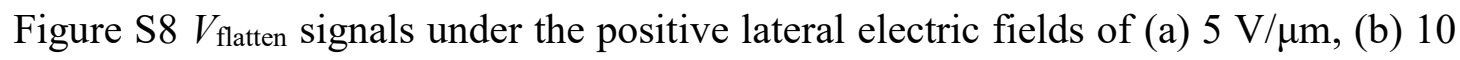
$\mathrm{V} / \mu \mathrm{m}$, (c) $15 \mathrm{~V} / \mu \mathrm{m}$, (d) $20 \mathrm{~V} / \mu \mathrm{m}$, (e) $25 \mathrm{~V} / \mu \mathrm{m}$ and (f) $30 \mathrm{~V} / \mu \mathrm{m}$. 


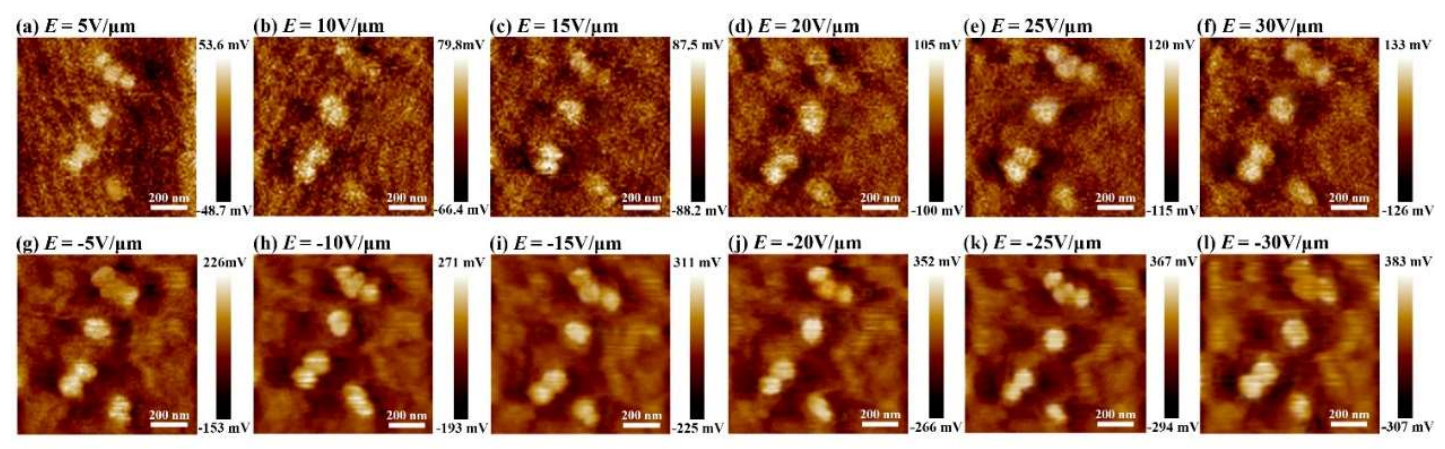

Figure S9 $V_{\text {flatten }}$ signals under the (a-f) positive and (g-l) negative lateral electric fields at the temperature of $60{ }^{\circ} \mathrm{C}$. 